\title{
La destinée d'Hymenolepis nana var. fraterna (Cestode) chez un hôte inhabituel : Leucophaea maderae (Dictyoptère)
}

\author{
par B. PESSON et N. LEGER \\ Laboratoire de Parasitologie, U.E.R. Pharmacie, \\ 4, avenue de l'Observatoire, F 75006 Paris
}

Le cysticercoïde d'Hymenolepis nana var. fraterna se développe normalement dans la cavité générale de divers Insectes: Coléoptères, Orthoptères, Siphonaptères, qui ne présentent aucune réaction apparente au parasitisme. Les Dictyoptères, en particulier Leucophaea maderae, se montrent réfractaires à l'infestation par la voie buccale. En revanche, lorsque des embryons hexacanthes sont introduits par effraction des téguments dans la cavité générale de ces insectes, ils sont capables de se développer jusqu'au stade cysticercoïde infestant. On observe alors d'importantes réactions : formation de capsules cellulaires et mélanisation, sans que la vitalité des vers en soit affectée (Cavier et Léger, 1965). D'autre part, il a été montré (Léger et Cavier, 1970) que le milieu intestinal de la Blatte ne s'oppose pas à l'éclosion de l'œuf : les embryons hexacanthes obtenus, transplantés dans la cavité générale de blattes neuves, s'y développent normalement. C'est donc au niveau de la paroi intestinale que se situe l'obstacle à l'infestation par les voies naturelles.

La destinée des embryons hexacanthes au cours de leur tentative de traversée de la paroi intestinale a été étudiée sur coupes histologiques chez Leucophaea maderae.

Des blattes mises à jeuner pendant 48 heures sont gavées individuellement avec des anneaux mûrs d'Hymenolepis nana et maintenues à $30^{\circ} \mathrm{C}$ (Voge et Turner, 1956 ; Voge et Heyneman, 1957). Le repas infestant étant pris comme temps 0, les blattes sont sacrifiées aux temps 1, 2, 24 heures et 2, 4, 6, 8, 10, 12, 15, 20 jours, et les intestins sont prélevés en vue de l'étude histologique.

Les coupes pratiquées à 1 heure et 2 heures environ montrent quelques embryons libres dans la lumière intestinale.

Sur les coupes de 24 heures, on observe la pénétration intra-épithéliale de très rares embryons hexacanthes. Certains sont dégénérés; d'autres intacts se situent déjà pour la plupart au niveau du conjonctif et des couches musculaires. 
Simultanément, se développent des réactions cellulaires de l'hôte se traduisant, d'une part, par une réaction péri-embryonnaire au niveau de l'épithélium, d'autre part, par un afflux d'hémocytes à la face externe de la paroi intestinale en regard de l'embryon. Cette dernière réaction semble bien être induite à distance puisque, au bout de 24 heures, l'embryon est encore inclus dans l'épaisseur de la paroi intestinale. Plus tard, ayant achevé son passage, il est retrouvé à l'intérieur de ces formations hémocytaires qui font hernie dans la cavité générale : il reste au stade hexacanthe et ne montre aucun s:gne d'évolution.

Dès le sixième jour, alors que l'embryon ne s'est toujours pas développé mais au contraire commence à montrer des signes de dégénérescence, on observe une diminution de la réaction cellulaire et l'apparition d'un dépôt de mélanine qui reste discret.

Comme le montrent les expériences d'infestation par la voie intra-cavitaire, il apparaît que la réaction hémocytaire seule n'entrave en rien le développement de l'embryon : le déclenchement du processus bloquant la croissance du parasite et entrâ̂nant sa dégénérescence est lié à la traversée de l'épithélium.

Des essais d'hyperinfestation par gavages répétés ont permis d'obtenir chez Leucophaea maderae (1 Blatte sur 10 expérimentées) une faible infestation qui s'est déroulée comme chez les insectes normalement réceptifs, sans réaction cellulaire.

Dans ce cas, il semblait que nous avions réussi à inonder complètement les processus de défense spécifique et aspécifique de l'hôte contre le parasite.

Nous avons tenté d'obtenir ce blocage en utilisant un autre procédé : l'irradiation par le Co 60. Des lots de blattes ont été soumises à des irradiations totales de 10000 et 20000 Roentgen puis infestées par des œufs d'H. nana (voie buccale) ou des embryons hexacanthes (voie intracavitaire).

L'infestation par la voie intracavitaire objective l'inhibition de la réaction hémocytaire : les cysticercoïdes se développent alors comme chez les hôtes naturels.

L'infestation par la voie buccale reste le plus souvent négative : sur 25 tentatives d'infestation, nous n'avons observé qu'une seule fois le développement de 3 embryons dans l'hémocoele.

Il semble bien que la réaction de l'hôte au parasite comporte deux temps distincts :

- un temps intestinal qui s'oppose au passage de la plupart des embryons et au développement ultérieur de ceux qui arrivent à franchir la paroi. Cette réaction persiste après irradiation. La nature, épithéliale ou hémocytaire, des cellules en cause reste à préciser en microscopie électronique. Une telle étude s'avère laborieuse du fait du nombre réduit des passages;

- un temps hémocytaire incapable à lui seul de bloquer le développement de l'embryon. Il débute très tôt, avant même que le parasite ait traversé la paroi intestinale et reste discret dans le cas de l'infestation buccale. Cette réaction est supprimée par irradiation de l'hôte. 


\section{Bibliographie}

Cavier (R.) et Léger (N.), 1965. - A propos de l'évolution d'Hymenolepis nana, variété fraterna chez des hôtes intermédiaires inhabituels. Ann. Parasitol. hum. comp., 40, 655-658.

LÉGER (N.), 1966. - Contribution à l'étude du cysticercoïde d'Hymenolepis nana, variété fraterna, vis-à-vis de ses hôtes et des médicaments. Thèse de Doctorat d'Etat en Pharmacie, Paris.

Léger (N.) et Cavier (R.), 1970. - A propos de l'évolution d'Hymenolepis nana, variété fraterna, chez des hôtes intermédiaires inhabituels II. Ann. Parasitol. hum. comp., 45, 195-201.

Voge (M.) et Heyneman (D.), 1957. - Development of Hymenolepis nana and Hymenolepis diminuta (Cestoda, Hymenolepididae) in the intermediate host Tribolium confusum, 59, 549-579, University of California Press, édit., Berkeley.

Voge (M.) et TURner (J. A.), 1956. - The effect of different temperatures on the development of Hymenolepis diminuta in Tribolium confusum. J. Parasitol., 42, 31 et Exp. Parasitol., 5, 580-586. 